\title{
Ultrafast Nanoscale Phase-Change Memory Enabled By Single-Pulse Conditioning
}

DOI:

10.1021/acsami.8b16033

\section{Document Version}

Accepted author manuscript

Link to publication record in Manchester Research Explorer

\section{Citation for published version (APA):}

Loke, D., Skelton, J., Lee, T. H., Zhao, R., Chong, T-C., \& Elliott, S. R. (2018). Ultrafast Nanoscale Phase-Change Memory Enabled By Single-Pulse Conditioning. ACS Applied Materials and Interfaces , 10(49), 41855-41870. https://doi.org/10.1021/acsami.8b16033

\section{Published in:}

ACS Applied Materials and Interfaces

\section{Citing this paper}

Please note that where the full-text provided on Manchester Research Explorer is the Author Accepted Manuscript or Proof version this may differ from the final Published version. If citing, it is advised that you check and use the publisher's definitive version.

\section{General rights}

Copyright and moral rights for the publications made accessible in the Research Explorer are retained by the authors and/or other copyright owners and it is a condition of accessing publications that users recognise and abide by the legal requirements associated with these rights.

\section{Takedown policy}

If you believe that this document breaches copyright please refer to the University of Manchester's Takedown Procedures [http://man.ac.uk/04Y6Bo] or contact uml.scholarlycommunications@manchester.ac.uk providing relevant details, so we can investigate your claim.

\section{OPEN ACCESS}




\title{
Ultrafast Nanoscale Phase-Change Memory Enabled By Single-Pulse Conditioning
}

\author{
Desmond K. Loke,,${ }^{1,}$ Jonathan M. Skelton, ${ }^{2}$ Tae-Hoon Lee, ${ }^{3}$ Rong Zhao, ${ }^{4}$ Tow-Chong \\ Chong, ${ }^{1}$ Stephen R. Elliott ${ }^{3, *}$
}

\begin{abstract}
${ }^{I}$ Department of Science and Math, Singapore University of Technology and Design, 8 Somapah Road, Singapore 487372, Singapore

${ }^{2}$ Department of Chemistry, University of Bath, Claverton Down, Bath BA2 7AY, UK

${ }^{3}$ Department of Chemistry, University of Cambridge, Lensfield Road, Cambridge CB2 1EW, UK

${ }^{4}$ Department of Engineering Product Development, Singapore University of Technology and Design, 8 Somapah Road, Singapore 487372, Singapore
\end{abstract}

Keywords: nanoscale, electric-field control, meta-material, thermal engineering, phase-change memory

\begin{abstract}
We describe how the crystallization kinetics of a suite of phase-change systems can be controlled by using a single-shot treatment via "initial crystallization" effects. Ultra-rapid and highly-stable phase-change structures (with excellent characteristics), viz. conventional and sub-10 nm-size cells (400 ps switching and $368 \mathrm{~K}$ for ten-year-data retention), stackable cells (900 ps switching and $10^{6}$ cycles for similar-'switching-on' voltages) and multi-level configurations (800 ps switching and resistance-drift power-law coefficients $<0.11)$ have been demonstrated. Material measurements and thermal calculations also reveal the origin of the pre-treatment-assisted increase in crystallization rates, and the thermal diffusion in chalcogenide structures, respectively.
\end{abstract}


Phase-change memory (PCM) is an excellent candidate for achieving a 'universal' memory structure. ${ }^{1,2}$ PCMs are based on the reversible transition between the amorphous and crystalline states of a chalcogenide material — showing a marked contrast in physical characteristics, e.g. optical reflectance and electrical conductivity, between the phasesof which the $\mathrm{Ge}_{2} \mathrm{Sb}_{2} \mathrm{Te}_{5}$ (GST-225) system is perhaps the best known. ${ }^{3-5}$ Crystalline GST-225 has also shown interesting thermoelectric properties. ${ }^{6}$ The amorphous-tocrystalline transition is often referred to as "set", and the crystalline-to-amorphous transition is termed "reset". However, the rate of crystal nucleation and growth is insufficiently fast compared to that of melting and quenching to the amorphous state, and this limits the overall switching time. Best-performing phase-change structures with archetypal configurations, viz. two-state $(0,1)$, have exhibited structural ordering using voltage pulses of durations on the order of 1-10 ns. ${ }^{7}$ It is questionable if these times are sufficient for 'universal' memory applications, cf. traditional RAM switching times are around 1 ns. $^{8}$ A difficulty arises from the trade-off between increasing the rate of crystallization and, at the same time, extending the stability of the amorphous phase for long-term data retention. ${ }^{9}$ This has prevented widespread PCM commercialization.

Recently, an incubation-based approach has been used to control crystal nucleation and growth kinetics to furnish crystallization times below 1 ns using conventional GST. ${ }^{10}$ However, such incubation-based systems capable of 500-picosecond crystallization pulses, using a constant low voltage (to produce thermal prestructural ordering) and with multiple low-voltage usage, need to be improved. This is important to 
meet the ever-rising demand for faster consumer electronics. This thermal-incubation process is very different from the model of Karpov et al., ${ }^{11}$ which assumes a direct electric-field-induced modification of the crystal-nucleation barriers in GST. Rao et al. recently pointed out that a not so conventional $\mathrm{Sc}$-doped $\mathrm{Sb}_{2} \mathrm{Te}_{3}$ phase-change material without pre-programming has a rapid switching time of 700 picoseconds. ${ }^{12}$

Melting (and quenching) in the central region of a memory cell can occur, followed by crystallization spatially separated from the centre (Figure 1a). The melting rate is faster at higher temperatures, while rapid crystal nucleation and growth occurs at lower temperatures. ${ }^{13}$ This can be produced by using a voltage pulse of high amplitude and moderate duration (hereafter referred to as a 'shot'.) (Figure 1b) The "melting-andcrystallization" state can show a similar resistivity to that of the bare "reset" state due to the formation of minute crystals spatially separated from the centre. The overall rates of crystal nucleation and growth are essentially increased because of the high surface-tovolume ratio of such minute structures. These crystallites can readily be preserved after reversible switching; the standard moderate-amplitude voltage pulse for "set" switching and high-amplitude voltage pulse for "reset" switching (Figure 1b) archetypally produces crystallization and melting at central regions, respectively. This will permit a 'single'usage, incubation-based methodology (Figure 1c). The "melting-and-crystallization" state provides a route to achieve a similar stability of the amorphous structure to that for the bare "reset" state, e.g. at annealing temperatures below melting and crystallization temperatures, since the crystal-growth rate is slower at lower temperatures. The meltingand-crystallization phenomenon has been similarly observed using GST-based thin films 
using laser pulses, which are excellent prospects for optical memory applications; however, the formation mechanism is still unknown. ${ }^{14}$ Zhou et al. have harnessed annealing to obtain a crystalline matrix, and subsequent melting and quenching to form an amorphous region embedded in crystalline templates using promising thin-film SiSbTe-based devices. ${ }^{15}$ However, due to crystallization designed to occupy an entire thin film, the surface-to-volume ratio is often insufficiently high; this has produced an inadequate increase in the rate of crystal nucleation and growth. In addition, BrightSky et al. have developed an interesting atomic-layer deposition (ALD) process which can deposit nano-crystalline GST within the pore of a device and the devices can exhibit high-resistance readings, but the formation mechanism remained unclear. ${ }^{16}$

Here, we control the crystallization rates with a single-shot treatment to produce thermal initial crystallization via Joule heating, which permits faster crystal nucleation and growth upon subsequent "set" pulses. A 400-picosecond crystallization pulse and an extrapolated ten-year data-retention temperature of $368 \mathrm{~K}$ were achieved for a GST system with an archetypal and below-10 $\mathrm{nm}$ structure. This represents the fastest of the current reported PCMs with conventional and sub-10 nm configurations under full recrystallization conditions, and with the use of the voltage-peak full-width-halfmaximum (FWHM) as a measure of the pulse duration. ${ }^{17,18}$ A 900-picosecond crystallization pulse and $10^{6}$ cycles of similar 'switching-on' voltage pulses were also demonstrated using a configuration with a stacked structure. Namely, this is the first demonstration of sub-1 ns switching of a PCM with stacked configurations under full recrystallization conditions, with the use of the voltage-peak FWHM as a measure of the 
pulse duration. ${ }^{19,20}$ A system with a multi-level structure also exhibited an 800picosecond structural-ordering time and resistance-drift power-law coefficients below 0.11. This is about an order of magnitude faster than the fastest switching times of any of the existing PCMs with multi-state configurations under full recrystallization conditions and with the use of the voltage-peak FWHM as a measure of the pulse duration. ${ }^{21,22}$ Material characterization and thermal modelling also elucidate the origin of the prepulsing-assisted increase in crystallization rate, and the thermal diffusion in PCM structures, respectively.

We have probed the electrical signatures for a suite of phase-change systems, viz. prototypical and sub-10-nanometre, multi-level and stacked configurations, mainly with GST-225 'via' pore-like structures, using our custom-built electrical testing system ${ }^{10}$ (see Experimental and Modelling Methods in Supporting Information). The archetypal configuration, for instance, comprised silicon-dioxide $\left(\mathrm{SiO}_{2}\right)$-on-Si as the starting structure, on which a $200 \mathrm{~nm}$-thick TiW bottom electrode was formed, followed by the patterning and etching of a $20 \mathrm{~nm}$-thick $\mathrm{SiO}_{2}$ insulating layer to form pores, which were filled with a $20 \mathrm{~nm}$-thick GST-225 active layer. Finally, a $200 \mathrm{~nm}$-thick TiW top electrode was deposited to complete the structure (Figure 1e). Devices were initially in the high-resistivity state. Some studies were of the 'set-and-cook' type, in which we chose to pre-treat the structures once by using a $\sim 5.0 \mathrm{~V}-60 \mathrm{~ns}$ voltage pulse. For example, this is about two times shorter in time than the shortest times used for previous studies under negligible crystallization conditions and with the use of the FWHM as a measure of the pulse duration. ${ }^{10}$ This is important to permit energy-efficient memory devices (see 
Detailed Electrical and Calculation Analysis in Supporting Information). This was followed by subsequent crystallization from the high- to low-resistance levels, viz. around $300 \mathrm{k} \Omega$ and $10 \mathrm{k} \Omega$, respectively (Figure 1c). The duration of the shortest pulse able to crystallize the systems was measured (Figure 1d). Configurations without prepulsing were characterized for comparison. We have also examined the effect of temperature on the stability of the system in the high-resistivity phase.

A key finding of the present work is that, whereas conventional systems without pre-pulsing did not show crystal nucleation and growth for voltage pulses with durations less than the DRAM limit of 1 ns, pre-treated structures exhibited crystallization when subject to a sub-nanosecond voltage pulse, viz. around $1.6 \mathrm{~ns}$ and $800 \mathrm{ps}$ at $\sim 1.0 \mathrm{~V}$ with 5 $\mathrm{nm}$ pore configurations for non-pretreated and pretreated systems, respectively (Figure 2a). For example, this is about two times faster for an increase in pre-treatment, and the smallest of current reported PCMs with pore-like structures under full recrystallization conditions. ${ }^{17}$ These findings were observed while the data retention for the amorphous phase was not affected, viz. 10 years extrapolated data retention at $\sim 368 \mathrm{~K}$ and $372 \mathrm{~K}$ for the systems with and without pre-pulses, respectively (Figure 2b). The pre-treated structures also showed reversible and stable switching between the amorphous and crystalline phases with voltage-pulse durations below $1 \mathrm{~ns}$ for 10,000 cycles using a $5 \mathrm{~nm}$ pore configuration, as well as faster crystallization, e.g. $\sim 400 \mathrm{ps,} \mathrm{with} \mathrm{an} \mathrm{increase} \mathrm{in} \mathrm{pre-}$ treatment-pulse length (Figures 2c,d). For instance, this is about $20 \%$ faster than the fastest times achieved by previous record studies under full recrystallization conditions and with the use of the FWHM as a measure of the pulse duration. ${ }^{10}$ The pretreated 
system can also show excellent characteristics in terms of time-dependent resistance drift for the amorphous phase, pore-size dependent crystallization time, endurance of the glassy state, crystalline-to-amorphous transition time and crystallization voltage (Figures 2e,f, see Figures S2b,c and Additional Electrical and Modelling Data in Supporting Information). Furthermore, the pretreated system with stacked configurations can exhibit superior properties in terms of crystallization time and stability of the pre-treated system in the low-resistance state, e.g. 900 ps switching and $10^{6}$ cycles for similar "switchingon" voltages, and the pretreated system with multilevel configurations can show outstanding signatures in terms of crystallization time and time-dependent drift of the resistance levels, e.g. 800 ps switching and resistance-drift power-law coefficients $<0.11$ (Figures 2g,h, see Figures S2a,4c and Additional Electrical and Modelling Data in Supporting Information). Such fast crystallization facilitated by pre-treatment is indicative of the ease of harnessing the pre-pulsing protocol for PCMs with different systems.

The observation of fast crystal nucleation and growth, facilitated by pre-treatment, therefore gives a clue as to the possible morphology of the system in the pre-pulsed state, namely that it is comprised of crystalline surfaces or structures surrounding an amorphous matrix of GST-225. The significance of ordered configurations for the structures in the pre-pulsed phase has also been noted from electric-field waveform studies, ${ }^{23}$ but the component of the pre-treated state, and its structure, has not hitherto been investigated. It is important to note that, by transmission electron-microscopy (TEM) inspection, the pre-treated configuration can show minute ordered structures 
(albeit in low concentration) around the glassy region, for instance, near the insulating or electrode layers (Figure 3a). The ordered structures can act as a template for crystal growth, i.e. with negligible nucleation, resulting in a fast crystallization rate, ${ }^{7}$ although this direction will be investigated in the future. The pre-pulsed configurations can also show similar crystalline structures after reversible switching (see Figure 3b). It is notable that the non-prepulsed configuration does not show any ordered structures throughout the entire cell (Figure 3c). The ordered structures can be preserved after many reset and set cycles, which is demonstrated from the TEM observations. This is why the effect of the initial pre-pulse can persist during such cycles. These ordered structures do not perniciously affect the thermal-stability properties, as shown in Figure $2 \mathrm{~b}$. We speculate that the thermal distribution can be that, during reset, the ordered structures do not completely disappear. This can be very different from the centre of the cell, which is exposed to very high temperatures during the reset, eventually removing the memory of pre-heating, although this will be investigated in future work. This means that amelioration of the crystallization time can be related to the local area near the edge of a cell. Furthermore, theoretical modelling reproduces the high temperatures required for crystal formation near the boundaries of the GST-225 layer at different ends or corners of the GST models, viz. top, right, top right, for both high excitation voltages and moderate durations, e.g. $\sim 600 \mathrm{~K}-700 \mathrm{~K}$ (see Figure 1a and Supporting Information Figure S5). The pre-treated systems can also show a low degree of crystallinity, and it is notable that, although we do not observe a pre-pulsed duration shorter than the RAM limit of 1 nanosecond, the pre-pulsing duration is reduced with an increase in pre-pulsed voltage, although experiments to use pre-treatment pulses below a nanosecond will be 
investigated in future studies (Figure 1b, see Other Electrical Data in Supporting Information).

It is likely that the voltage and pulse length can influence the type and volume of ordered structures, which can subsequently affect the crystallization time. Experimentally, it can be generally difficult to directly observe the effect of voltage and pulse length on the type and volume of ordered structures, and the effects of type and volume of ordered structures on the subsequent crystallization time. However, our pretreatment pulse-length experiment is likely to provide some insight. It can be noted that the cell shows a pre-treatment pulse-length dependent crystallization time (Figure 2d). On the one hand, when the pre-treatment pulse is shorter than or equal to around $40 \mathrm{~ns}$, the cell can show a crystallization time that is not so different from those of a cell without pre-treatment. It can be that the small or no influence is related to the possibility that the volume of the ordered structure is negligible or absent. On the other hand, when the pretreatment pulse is longer than around $40 \mathrm{~ns}$, the cell can show a decreased crystallization time with an increase in pre-treatment-pulse length. This finding indicates that a pretreatment pulse longer than $\sim 40 \mathrm{~ns}$ might be long enough to produce a sufficient volume of ordered structures, so that the crystallization time can be effectively reduced, although future work should be carried out to investigate this effect, including the dependence of the crystallization time or volume of ordered structures on the pre-treatment voltage value, and the influence of the type of ordered structures on the crystallization time, whereby these trends can be similar. 
In our switching experiments, the sub-10 nm-sized cell ( $5 \mathrm{~nm}$-wide pore) without pre-treatment can show a crystallization time of around $1.6 \mathrm{~ns}$ (Figure 2a), in contrast to the same-sized cell with pre-treatment which shows a crystallization time of $\sim 400$ ps (Figure 2d), i.e. approximately four times shorter compared to the non-pretreated cell. It can be that the crystallization time is always noticeably reduced for the pre-treated cell compared to the non-pretreated cell with the same cell size (Figure 2f, inset). Therefore, even for a very small-sized device, we expect that further improvement can be achieved with pre-treatment in addition to the improvement by scaling alone; so that the best performance can be realized by downscaling with pre-treatment, but without significant degradation of the thermal stability (Figure 2b), which is another requirement for highdensity memory.

Thus, the results suggest that rapid, stable, and nanometer-scale switching operations could be produced using conventional, stacked and multi-level systems with a single-shot pre-treatment. By using structures with a higher proclivity for nucleation and growth and, at the same time, showing robustness to spontaneous crystallization, a systematic increase in the rate of switching can be achieved while maintaining a high stability of the amorphous state.

Our work demonstrates fast-switching speed but highly-stable phase-change structures for conventional and sub-10 nm size (viz. $400 \mathrm{ps}$ switching, $368 \mathrm{~K}$ for ten-year data retention), stackable cells (e.g. 900 ps switching, $10^{6}$ cycles for similar 'switchingon' voltages) and multi-level configurations (i.e. 800 ps switching, resistance-drift power- 
law coefficients $<0.11)$ for a single-shot pulse pre-treatment. This pre-treatment method, well suited to the study of the creation of fully ordered minute structures, should be a complementary, and cost-effective, alternative technique to established electrically-based methods, involving field aspects of crystallization, for the rapid optimization of configurations suitable for phase-change memories.

\section{Associated Content}

\section{Supporting Information}

The Supporting Information is available free of charge on the ACS Publication Website at...

Details of Experimental and Modelling Methods, Additional Electrical and Modelling Data, Detailed Electrical and Calculation Analysis, Other Electrical Data, and Data of Electrical Characterization and Thermal Modelling.

\section{Author Information}

\section{Corresponding Authors}

Email: desmond_loke@sutd.edu.sg

Email:sre1@cam.ac.uk

\section{Notes}

The authors declare no competing financial interest.

\section{Acknowledgements}

The authors thank W.J. Wang, H.X. Yang, K.G. Lim, L.T. Ng, L.T. Law, L. Li and S.S.Y. Chan for valuable contributions on the electrical-characterization and theoretical-calculation studies. 
We acknowledge financial support by the Engineering and Physical Sciences Research Council (United Kingdom). We thank the Agency for Science, Technology and Research (Singapore) for the use of their facilities to fabricate and characterize the devices. D.K.L. thanks the SUTD and MOE Grant Programs for research support. 


\section{References}

1) Wuttig, M., 2005. Phase-Change Materials: Towards a Universal Memory?. Nat. Mater. 2005, 4, 265-266.

2) Perniola, L.; Molas, G.; Navarro, G.; Nowak, E.; Sousa, V.; Vianello, E.; De Salvo, B. Universal Signatures from Non-Universal Memories: Clues for the Future. Proc. IEEE 8th International Memory Workshop 2016, 1-3. DOI: 10.1109/imw.2016.7495295.

3) Hosseini, P.; Wright, C. D.; Bhaskaran, H. An Optoelectronic Framework Enabled by Low-Dimensional Phase-Change Films. Nature 2014, 511, 206-211.

4) Y. Shi, S. Fong, H. S. P. Wong, D. Kuzum, Neuro-Inspired Computing using Resistive Synaptic Devices, Springer, Gewerbestrasse, Cham, Switzerland 2017.

5) Xie, Y.; Kim, W.; Kim, Y.; Kim, S.; Gonsalves, J.; BrightSky, M.; Lam, C.; Zhu, Y.; Cha, J. J. Self-Healing of a Confined Phase Change Memory Device with a Metallic Surfactant Layer. Adv. Mater. 2018, 30, 1705587-1-7.

6) Suh, D. S.; Kim, C.; Kim, K. H.; Kang, Y. S.; Lee, T. Y.; Khang, Y.; Park, T. S.; Yoon, Y. G.; Im, J.; Ihm, J. Thermoelectric Heating of $\mathrm{Ge}_{2} \mathrm{Sb}_{2} \mathrm{Te}_{5}$ in Phase Change Memory Devices. Appl. Phys. Lett. 2010, 96, 123115-1-3.

7) Bruns, G.; Merkelbach, P.; Schlockermann, C.; Salinga, M.; Wuttig, M.; Happ, T. D.; Philipp, J. B.; Kund, M. Nanosecond Switching in GeTe Phase Change Memory Cells. Appl. Phys. Lett. 2009, 95, 043108-1-3.

8) U. Schroeder, K. Cho, S. Slesazeck, Thin Films on Silicon: Electronic and Photonic Applications, World Scientific, Singapore, Singapore 2016.

9) Chen, Y.; Wang, G.; Li, J.; Shen, X.; Xu, T.; Wang, R.; Lu, Y.; Wang, X.; Dai, S.; Nie, Q. Sb-Rich Zn-Sb-Te Phase-Change Materials: A Candidate for the Trade-Off between Crystallization Speed and Data Retention. Appl. Phys. Express 2014, 7, 105801-1-4. 
10) Loke, D.; Lee, T. H.; Wang, W. J.; Shi, L. P.; Zhao, R.; Yeo, Y. C.; Chong, T. C.; Elliott, S. R. Breaking the Speed Limits of Phase-Change Memory. Science 2012, 336, 1566-1569.

11) Karpov, V. G., Kryukov, Y. A., Karpov, I. V. Mitra, M. Field-Induced Nucleation in Phase Change Memory. Phys. Rev. B 2008, 78, 052201-1-4.

12) Rao, F.; Ding, K.; Zhou, Y.; Zheng, Y.; Xia, M.; Lv, S.; Song, Z.; Feng, S.; Ronneberger, I.; Mazzarello, R.; Zhang, W. Reducing the Stochasticity of Crystal Nucleation to Enable Subnanosecond Memory Writing. Science, 2017, 358, 1423-1427.

13) Orava, J.; Greer, A. L.; Gholipour, B.; Hewak, D. W.; Smith, C. E. Characterization of Supercooled Liquid $\mathrm{Ge}_{2} \mathrm{Sb}_{2} \mathrm{Te}_{5}$ and its Crystallization by Ultrafast-Heating Calorimetry. Nat. Mater. 2012, 11, 279-283.

14) Chu, C. H.; Da Shiue, C.; Cheng, H. W.; Tseng, M. L.; Chiang, H. P.; Mansuripur, M.; Tsai, D. P. Laser-Induced Phase Transitions of $\mathrm{Ge}_{2} \mathrm{Sb}_{2} \mathrm{Te}_{5}$ Thin Films used in Optical and Electronic Data Storage and in Thermal Lithography. Opt. Express 2010, 18, 1838318393.

15) Zhou, X.; Wu, L.; Song, Z.; Rao, F.; Liu, B.; Yao, D.; Yin, W.; Feng, S.; Chen, B. Investigation of Sb-Rich $\mathrm{Si}_{2} \mathrm{Sb}_{2+\mathrm{x}} \mathrm{Te}_{6}$ Material for Phase Change Random Access Memory Application. Appl. Phys. A 2011, 103, 1077-1081.

16) Sky, M. B.; Sosa, N.; Masuda, T.; Kim, W.; Kim, S.; Ray, A.; Bruce, R.; Gonsalves, J.; Zhu, Y.; Suu, K.; Lam, C. Crystalline-As-Deposited ALD Phase Change Material Confined PCM Cell for High Density Storage Class Memory. Proc. IEEE Int. Electron Devices Meet. 2015, 60-63. DOI: 10.1109/IEDM.2015.7409621

17) Im, D. H.; Lee, J. I.; Cho, S. L.; An, H. G.; Kim, D. H.; Kim, I. S.; Park, H.; Ahn, D. H.; Horii, H.; Park, S. O.; Chung, U. I. A Unified 7.5 nm Dash-Type Confined Cell for High Performance PRAM Device. Proc. IEEE Int. Electron Devices Meet. 2008, 1-4. DOI: 
10.1109/iedm.2008.4796654

18) Xiong, F.; Liao, A.D.; Estrada, D.; Pop, E. Low-Power Switching of Phase-Change Materials with Carbon Nanotube Electrodes. Science, 2011, 332, 568-570

19) Kau, D.; Tang, S.; Karpov, I. V.; Dodge, R.; Klehn, B.; Kalb, J. A.; Strand, J.; Diaz, A.; Leung, N.; Wu, J.; Lee, S. A Stackable Cross Point Phase Change Memory. Proc. IEEE Int. Electron Devices Meet. 2009, 617-620. DOI: 10.1109/iedm.2009.5424263

20) Takaura, N.; Kinoshita, M.; Tai, M.; Ohyanagi, T.; Akita, K.; Morikawa, T. A 4F2Cross-Point Phase Change Memory using Nano-Crystalline Doped GeSbTe Material. Jpn. J. Appl. Phys. 2015, 54, 04DD01-1-6.

21) Athmanathan, A.; Stanisavljevic, M.; Papandreou, N.; Pozidis, H.; Eleftheriou, E. Multilevel-Cell Phase-Change Memory: A Viable Technology. Journal on Emerging and Selected Topics in Circuits and Systems 2016, 6, 87-100.

22) Rao, F.; Song, Z.; Zhong, M.; Wu, L.; Feng, G.; Liu, B.; Feng, S.; Chen, B. Multilevel Data Storage Characteristics of Phase Change Memory Cell with Doublelayer Chalcogenide Films $\left(\mathrm{Ge}_{2} \mathrm{Sb}_{2} \mathrm{Te}_{5}\right.$ and $\left.\mathrm{Sb}_{2} \mathrm{Te}_{3}\right)$. Jpn. J. Appl. Phys. 2007, 46, L25-L27.

23) Kang, D. H.; Young Kim, N.; Jeong, H.; Cheong, B. K. Understanding on the CurrentInduced Crystallization Process and Faster Set Write Operation thereof in Non-Volatile Phase Change Memory. Appl. Phys. Lett. 2012, 100, 063508-1-3. 


\section{Figure Captions}

Figure 1. Single-pulse pre-crystallization effects on the crystal nucleation and growth of phase-change systems. a) Finite-element-method simulations of the temperature distribution in the structure with low (left panel) and high (centre panel) excitation voltages. The pulse duration was kept constant, and the systems were excited by low (around $3.0 \mathrm{~V})$ and high $(\sim 5.0 \mathrm{~V})$ pulse voltages, respectively. Right panel: temperature profile of the GST-225 layer with high (red line) and low (blue line) excitation voltages, showing a lower rate of temperature reduction away from the GST225 centre with an increase in the excitation voltage. b) System resistance (colour scale) as a function of pulse voltage and pulse duration for the structure with an initially highresistance level. The structure of the system was kept constant (prototypical type), and the sizes of the pores of the configuration were maintained to be the same at around 50 nm. c) Illustration of current work for a voltage pulse with a high amplitude and moderate pulse length for single-pulse conditioning, with a short main pulse used to crystallize the configuration. The single conditioning pulse is harnessed for initiating crystal formation around the glassy material, followed by the main pulse, viz. in (d), to induce structural ordering of the system. e) The PCM structure, along with a photograph of the device and an atomic-force-microscope image of the pore, used for crystallizing the system (see Supporting Information Figure S1).

Figure 2: Pre-treatment dependence of the crystallization rate and amorphousphase stability of chalcogenide structures. a) Electrical voltage as a function of pulse 
duration needed for crystallizing phase-change memory cells with a conventional configuration (Figure 1e) with (purple line) and without (yellow line) pre-pulsing. b) An Arrhenius extrapolation for 10-year data retention (of the amorphous state) of the prototypical structure with (green line) and without (orange line) pre-pulsing. Supporting Information Figure S2f also shows the Arrhenius extrapolation for 10-year data retention obtained from experiments carried out on a different structure, and this structure also showed similar signatures. c) Plot of the resistance level as a function of the cycle number of a phase-change structure with pre-treatment with constant voltage pulses, e.g. high- to low-resistance levels, around $1.0 \mathrm{~V}, 800 \mathrm{ps}$; low- to high-resistance levels, about 5.0 V, 500 ps. d) Plot of crystallization-pulse duration as a function of pre-treatmentpulse duration for the pre-treated structure. The crystallization- and pre-treatment-pulse voltages were kept constant at around $1.0 \mathrm{~V}$ and $5.0 \mathrm{~V}$, respectively. e) Time evolution of the resistance level of the archetypal system with and without pre-pulsing (blue and pink lines), with a resistance-drift coefficient, $v$, determined by a fit to a power law. The ambient temperature was kept constant at around $300 \mathrm{~K}$. f) Pore-size dependence of pulse length with pre-treatment for crystallizing the structure under a constant pulse voltage $(\sim 1.0 \mathrm{~V})$. The inset shows a table of the pulse length for crystallizing the pre-treated system and the non-pretreated system and with different pore sizes, showing a decreased crystallization time for the pretreated structure compared to the non-pretreated structure for the same pore size. g) Correlation between the voltage amplitude and pulse duration with (blue bars) and without (orange bars) pre-pulsing to crystallize the system with a stacked configuration for constant pore size (about $35 \mathrm{~nm}$ ). The right panel shows the configuration of the structure. The systems were switched between high- and low- 
resistance levels of about $350 \mathrm{k} \Omega$ and $190 \mathrm{k} \Omega$, respectively, which are higher than the values for the conventional structure (around $300 \mathrm{k} \Omega$ and $10 \mathrm{k} \Omega$ ), owing to an additional switching layer in the active region of the device. h) Resistance (colour scale) as a function of voltage and for varying pulse durations with and without (right and left panels) pre-treatment to crystallize the structure with a multi-level configuration. The systems were switched between the high-, intermediate- and low-resistance levels of about $300 \mathrm{k} \Omega, 150 \mathrm{k} \Omega$ and $10 \mathrm{k} \Omega$, respectively. The coloured boxes show the average of the values obtained from experiments carried out on three different structures. The structures for (a, b, c, d, e, f, h) were initially in the high-resistance level $(\sim 300 \mathrm{k} \Omega)$, and the sizes of the structures for $(\mathrm{a}, \mathrm{b}, \mathrm{c}, \mathrm{d}, \mathrm{e}, \mathrm{h})$ were maintained to be the same at around 5 $\mathrm{nm}$. The structure with pre-treatment for $(\mathrm{a}, \mathrm{b}, \mathrm{c}, \mathrm{e}, \mathrm{f}, \mathrm{g}, \mathrm{h})$ was subjected to a voltage pulse (around $5.0 \mathrm{~V}, 60 \mathrm{~ns}$ ) in a single-pulse conditioning, while the non-treated system remained untouched. The error bars show the range of values obtained from experiments carried out on three different structures.

Figure 3. Pre-treated PCM morphology. a) Transmission-electron-microscope (TEM) images of the system with pre-pulsing, showing clusters are formed around the amorphous matrix when pre-treatment is used. b) TEM image obtained from experiments carried out on a different structure (after reversible switching). The structure also showed similar morphologies. c) TEM image of the system without pre-pulsing. The nonprepulsed system does not show any ordered structures throughout the entire cell, although part of the cell is shown in the figure. For (a-c), the device structure was kept constant (prototypical type), and the pore size of the system was fixed at around $50 \mathrm{~nm}$. 
The voltage amplitude and pulse duration for the pre-treatment were kept constant at approximately $5.0 \mathrm{~V}$ and $60 \mathrm{~ns}$, respectively. The system was initially in the highresistance state of about $300 \mathrm{k} \Omega$. The yellow dotted regions indicate the ordered structural regions. 


\section{Figures}

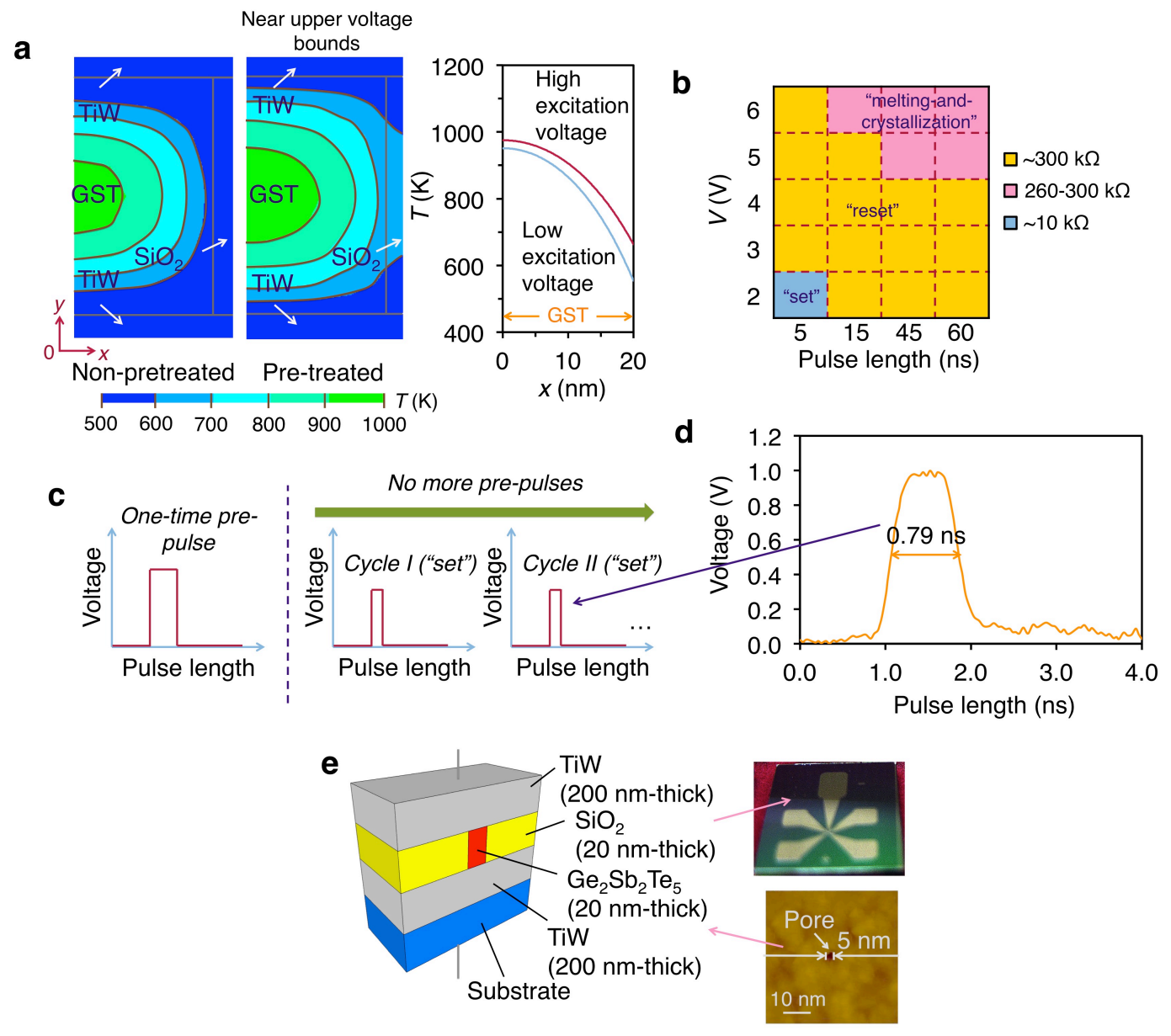

Figure 1. 

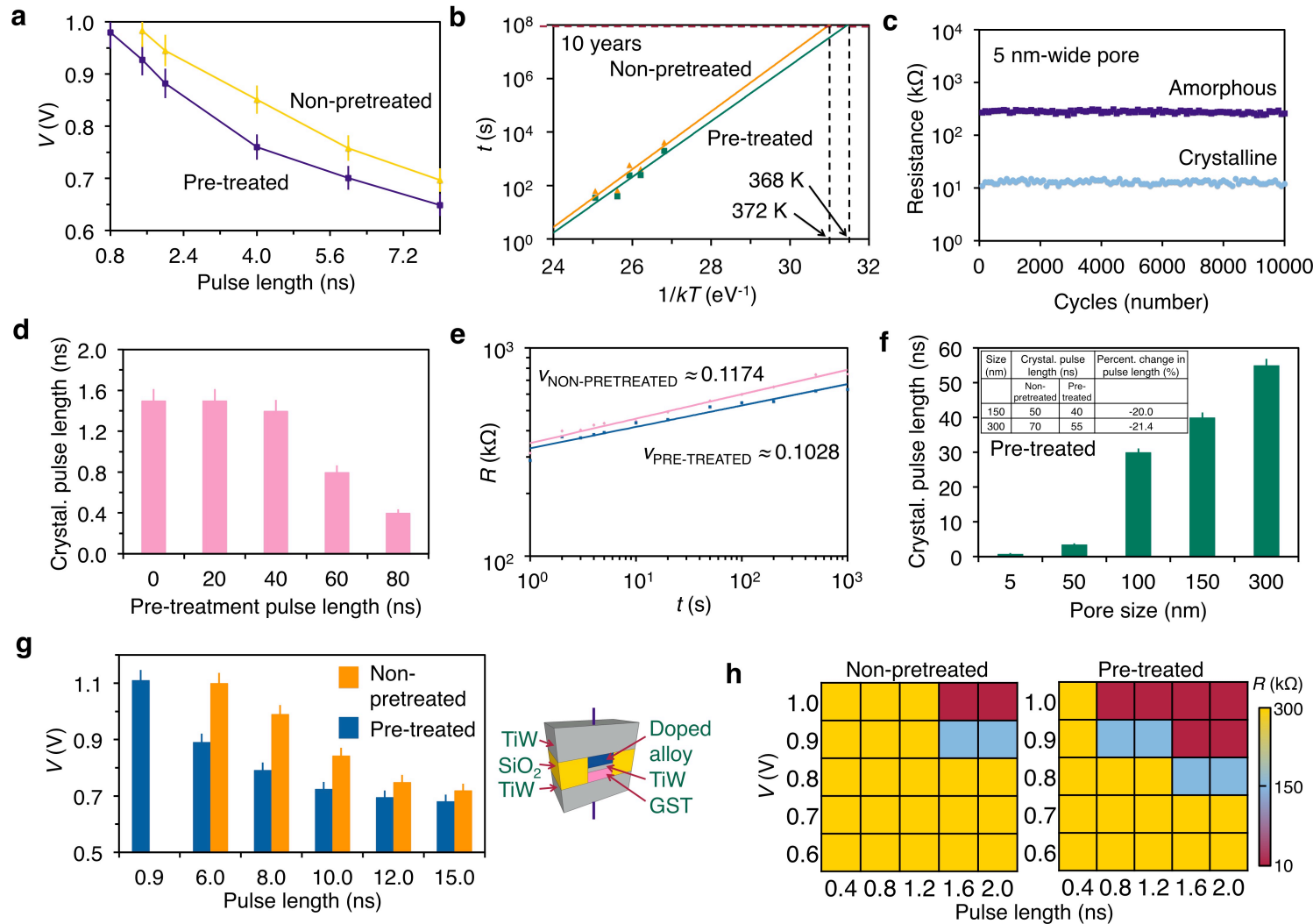

Figure 2. 

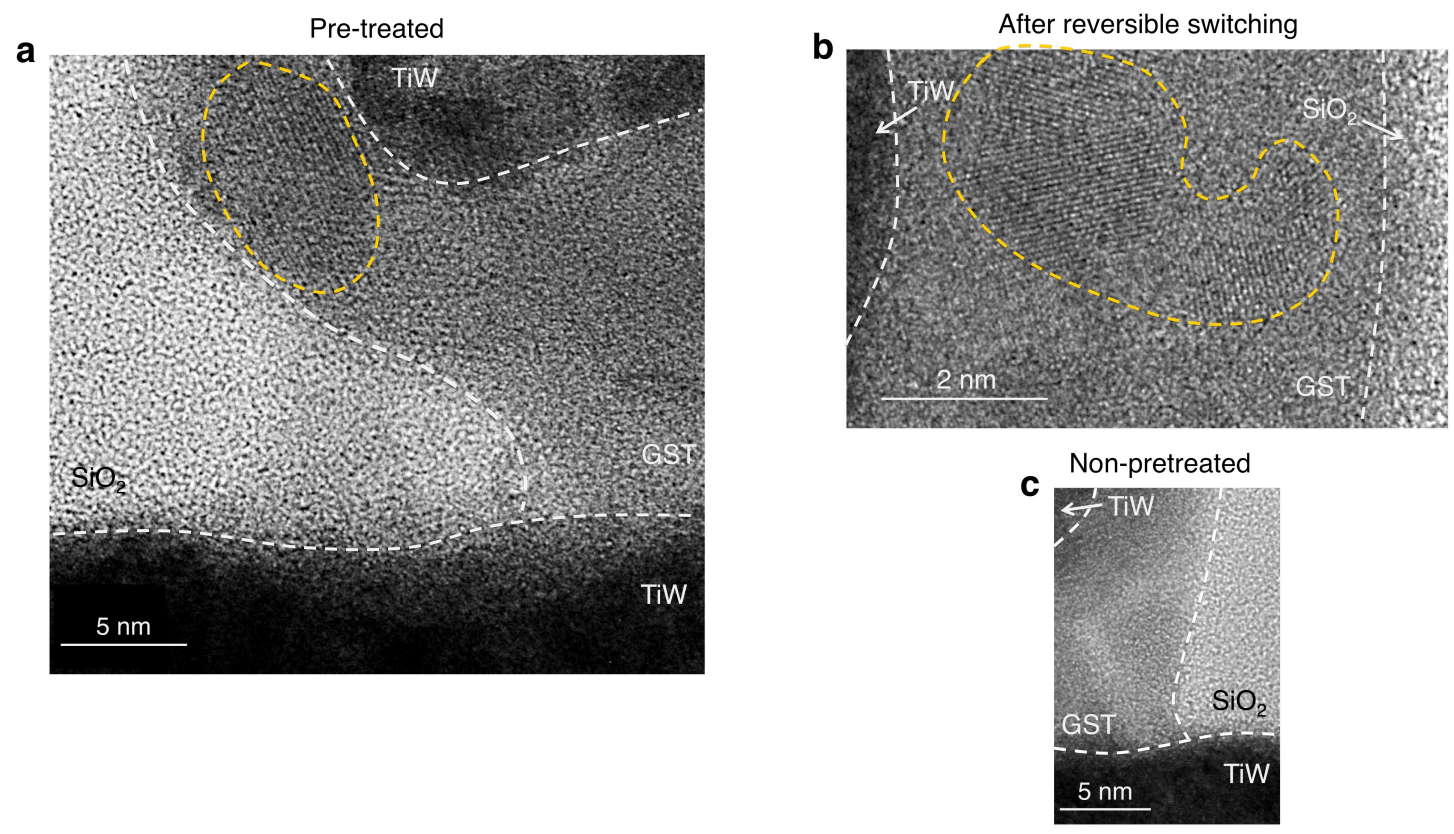

Figure 3. 


\section{ToC figure}
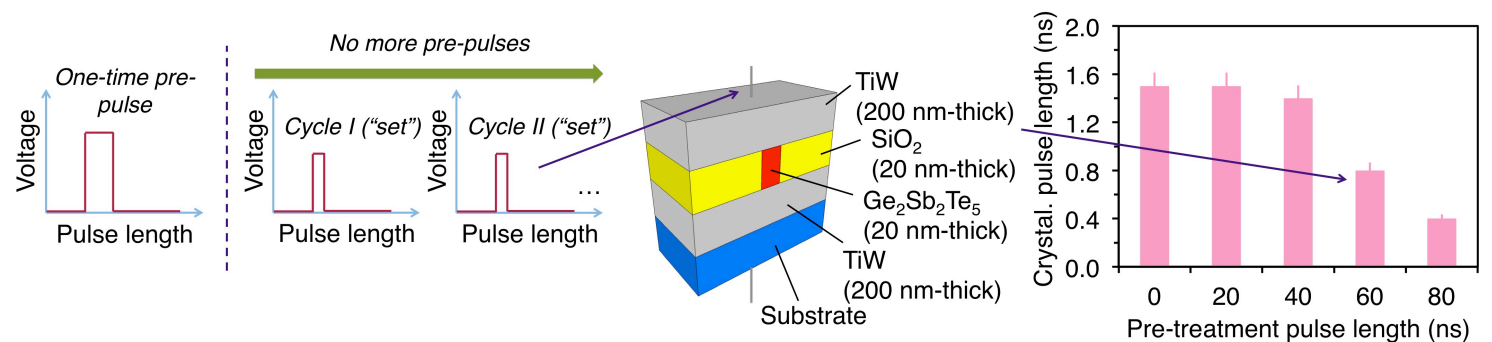